Special Issue of the 8th International Advances in Applied Physics and Materials Science Congress (APMAS 2018)

\title{
An Optimized and Logic Gate with High Transmission and Low Losses Based on Y-junction
}

\author{
R. Derdour*, M.R. Lebbal, A. Benmerkhi, T. Bouchemat, \\ M. Bouchemat And F. Mirouh \\ University of Brothers Mentouri Constantine 1, Department of Electronics, Laboratory L.M.I, Algeria \\ We propose the optimization of a micro-component for the integrated optics which is the AND logic gate \\ formed by a Y-junction based on two-dimensional crystal Photonics with a triangular lattice of air holes in silicon. \\ We chose this structure to improve the transmission of single mode with high power and low losses compared to \\ the AND logic gate classic, by optimizing the photonic crystal geometric parameters, the band gaps wavelength \\ and eliminate the higher order modes in order to realize an optimized AND logic gate.
}

DOI: 10.12693/APhysPolA.135.782

PACS/topics: photonic crystal, Y-junction, cavity, single mode, transmission, AND logic gate

\section{Introduction}

Photonic crystals are nanostructures consisting of materials with different refractive indexes arranged with light wavelength-scale periodicities. The periodicity results in certain forbidden bands for light, which is called photonic band gaps (PBGs), and light within the gap range is not allowed to propagate in the structures. Since a defect in the periodic structure can generate the allowed modes in the PBG region, point and line defects are selectively formed for realization of nano-cavities and waveguides, respectively [1-4]. In particular, due to advanced fabrication techniques of semi-conducts, semiconductorsbased photonic crystals have been utilized to achieve high-Q factor nano-cavities, low-loss structures and wide band waveguides.

In recent years, the use of photonic crystals as optical conductors in the transmission bands attracted many research efforts. The introduction of elements based on photonic crystal would treat all information in light and miniaturize the circuits. This is why many proposals with photonic crystal devices were produced and sometimes already completed and implemented in integrated optics such as waveguides, bends, filters, etc.

Among the advantages of integrated optics is to combine several optical functions on a single substrate. The advantages are stability, self-alignment of functions and reduction in size. Integrated optics may be carried on other materials with different technologies like for microelectronics.

Currently the optical logic gates have received much attention for their applications in real time optical processing and information communications [5], such as, AND logic gate is used to perform address recognition, packet header modification, and data integrity verification,

*corresponding author; e-mail: maroma18@yahoo.fr and as a sampling gate in optical sampling oscilloscope owing to their ultrafast operation compared to the traditional electrical methods $[6,7]$.

In this paper we aim to develop a basic element for integrated optics, the AND logic gate formed by Y-junction and two microcavities with a triangular lattice of air holes in silicon. The device studied is widely used in future optical microelectronics. The FDTD method has been used to simulate optical propagation in this junction. Two holes have been located in the intersection of the input and output channel waveguides. Their size has been varied in order to optimize the transmitted power and reduce the losses.

To validate our results numerically, we use a finitedifference time-domain (FDTD-2D) method to simulate the wave propagation inside the double bend and the $\mathrm{Y}$-junction splitter in a two-dimensional photonic crystal [8-10].

\section{Two dimensional FDTD method}

In the presence of non-vanishing conductivity, the 2D FDTD time stepping formulas for the TE modes are $[11,12]$.

$$
\begin{gathered}
\left.H_{z}\right|_{i, j} ^{n+1 / 2}=\left.H_{z}\right|_{i, j} ^{n-1 / 2} \\
+\frac{\Delta t}{\mu}\left(\frac{\left.\left.E_{x}\right|_{i, j+\frac{1}{2}} ^{n}-\left.E_{x}\right|_{i, j-\frac{1}{2}} ^{n}-\frac{\left.E_{y}\right|_{i+\frac{1}{2}, j} ^{n}-\left.E_{y}\right|_{i-\frac{1}{2}, j} ^{n}}{\Delta y}\right)}{\Delta y}\right) \\
\left.E_{x}\right|_{i, j} ^{n+1}=\left.E_{x}\right|_{i, j} ^{n}+\frac{\Delta t}{\varepsilon_{i, j}} \frac{\left.H_{z}\right|_{i, j+\frac{1}{2}} ^{n+\frac{1}{2}}-\left.H_{z}\right|_{i, j-\frac{1}{2}} ^{n+\frac{1}{2}}}{\Delta y} \\
\left.E_{y}\right|_{i, j} ^{n+1}=\left.E_{y}\right|_{i, j} ^{n}-\frac{\Delta t}{\varepsilon_{i, j}} \frac{\left.H_{z}\right|_{i+\frac{1}{2}, j} ^{n+\frac{1}{2}}-\left.H_{z}\right|_{i-\frac{1}{2}, j} ^{n+\frac{1}{2}}}{\Delta x}
\end{gathered}
$$


The index $n$ denotes the discrete time step, indices $i$ and $j$ denote the discretized grid point in the $x-y$ planes, respectively. The FDTD time stepping is given by:

$$
\Delta t \leq \frac{1}{c \sqrt{\left(\frac{1}{\Delta x}\right)^{2}+\left(\frac{1}{\Delta y}\right)^{2}}},
$$

where $c$ is the speed of light, to satisfy the numerical stability condition and $\Delta x$ and $\Delta y$ are the intervals between two neighboring grid points along the $x$ - and $y$-directions in the $x, y$ coordinate system, respectively.

With:

$$
\Delta x=\Delta y \leq \frac{\lambda}{10 \sqrt{\varepsilon_{r}}}
$$

\section{Operation principle and structure analysis}

The structure has formed by a Y-junction, which is the basic building block of integrated optics coupled with two microcavity introduced in the center of the three waveguide as shown in Fig. 1. This structure based on a triangular lattice of air holes in silicon, which has been chosen because triangular lattices may exhibit large band gaps and the silicon is expected to be a good platform for integrated photonics circuits and ultra-compact optical devices [8, 13].

In this structure, we present a study of triangular lattice crystal photonic of air holes in silicon. The refractive silicon index (3.42). The radius hole is selected as $r=0.4 a$, where $a$ is the lattice constant and $r$ is the radius of air holes were chosen for a triangular lattice to obtain a photonic band gap (PBG) around $1.55 \mu \mathrm{m}$ exist for the telecom wavelengths.

We have used a two-dimensional FDTD code that captures the simulation parameters (spatial discretization step, simulation mode (TE/TM), number of iterations), the injection conditions (injection of a guided mode through a Huygens surface) and the boundary condition type (wall, symmetric or anti-symmetric).

This crystal is lit by a Gaussian wave under normal incidence with a transverse electric (TE) polarized mode. The length of the photonic crystal is 19a and the time

\section{Port A}

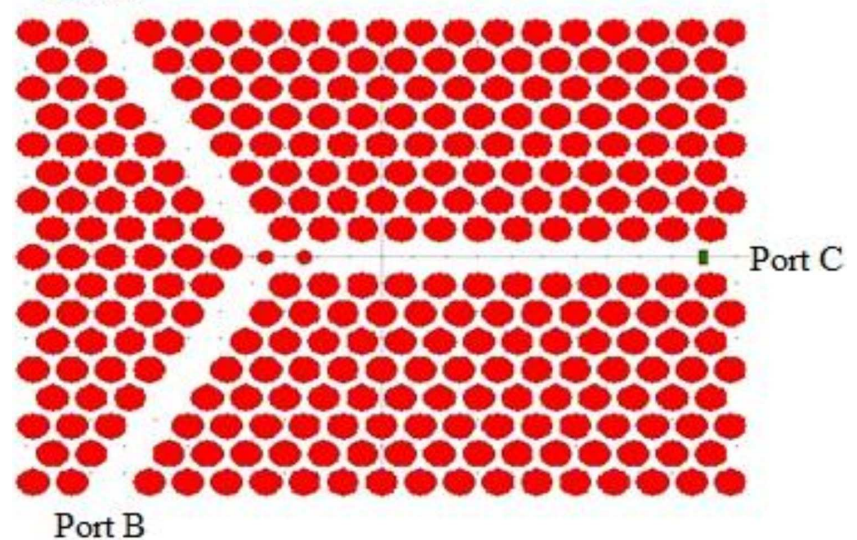

Fig. 1. Structure of the optimized AND logic gate. step is chosen equal to 0.01 . Note that it might be necessary to reduce the time step below the stability limit when simulating materials.

\section{Numerical analysis and discussion}

\subsection{Dispersion diagram}

Dispersion diagram showing normalized frequency versus the wave vector for transverse magnetic (TM) mode of the 2D photonic crystal is given in Fig. 2 .

It has been calculated by employing a $2 \mathrm{D}$ plane wave expansion method. A complete photonic band gap centered at telecommunication wavelengths can be determined. It runs between the normalized frequencies $\omega_{1}=0.25 a / \lambda$ and $\omega_{2}=0.40 a / \lambda$ which corresponds to wavelength range $1.4-2.4 \mu \mathrm{m}$ for the wave with TM polarization.

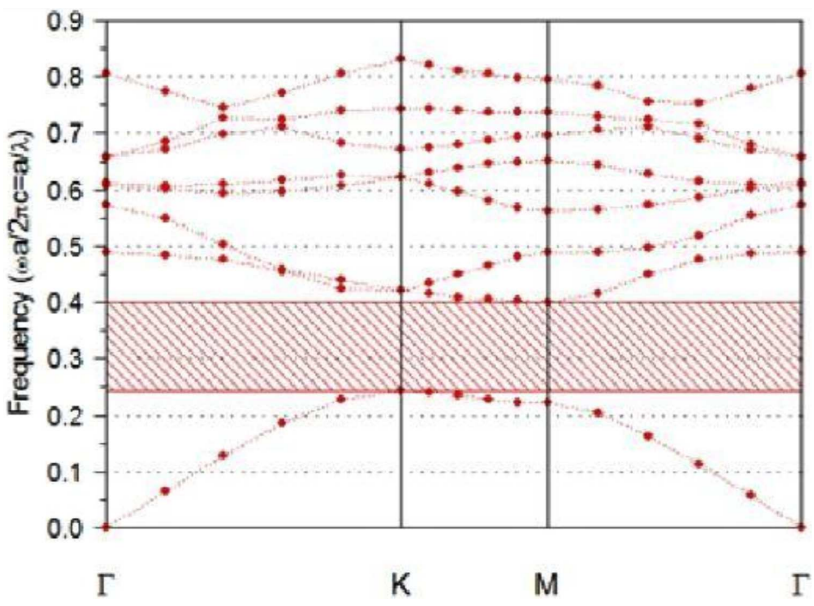

Fig. 2. Band gap structure of the photonic crystal layout.

\subsection{The transmitted power in the AND logic gate}

In this section, we analyze two different configurations. To determine $\mathrm{PhC}$ transmission, we need to specify wavelength range within which microcavities will be working.

\subsubsection{Before optimization}

Let us consider the 2D photonic crystal structure with a triangular lattice of air holes, the $\mathrm{Y}$-junction waveguide is formed and a hole is introduced at the center, this structure is excited with TM polarization. A pulsed Gaussian source is used to excite the fundamental waveguide mode at the waveguide input (Fig. 3).

Figure 4 represents the normalized transmission spectra with arbitrary unit obtained by the two-dimensional finite difference time domain (2D FDTD) simulation for the structure illustrated in Fig. 3.

The results of the 2D FDTD simulation shows clearly the low transmission, we also recorded a weak transmission with maximum of about 60 . This explains that there is a bad guided modes in this AND structure due to losses at the two corners. 
Port A

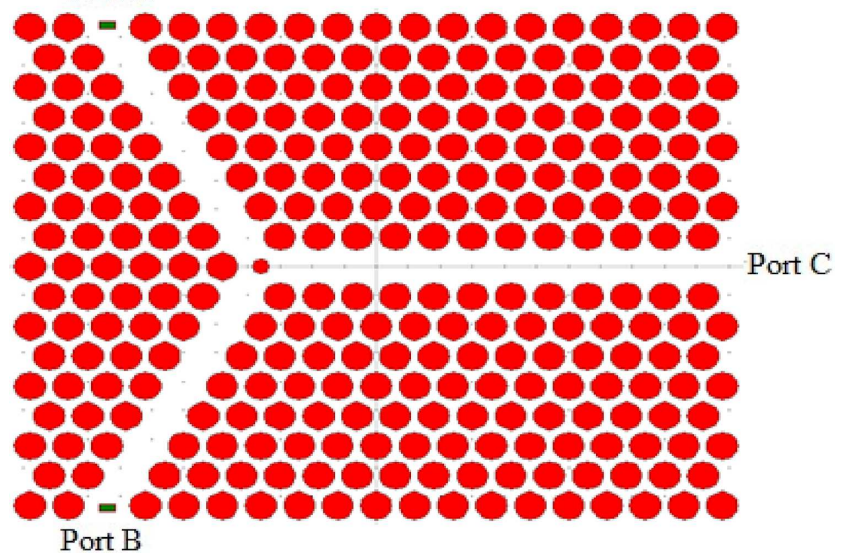

Fig. 3. Structure of AND logic gate with one microcavity.

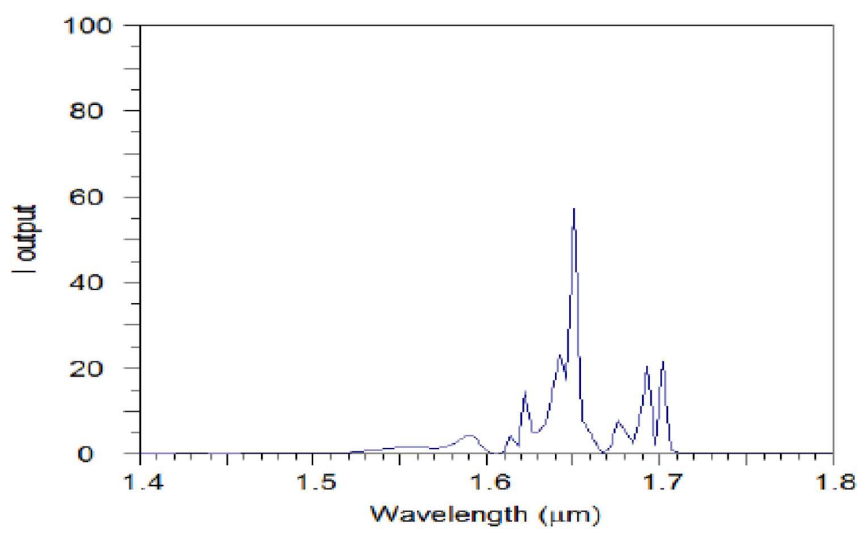

Fig. 4. The spectral response in transmission of the AND logic gate with one microcavity.

\subsubsection{After optimization}

This optimization keeps the same topology as compared to that of Fig. 3, with the exception to the addition of another microcavity in the center of $\mathrm{PhC}$ waveguides Fig. 1.

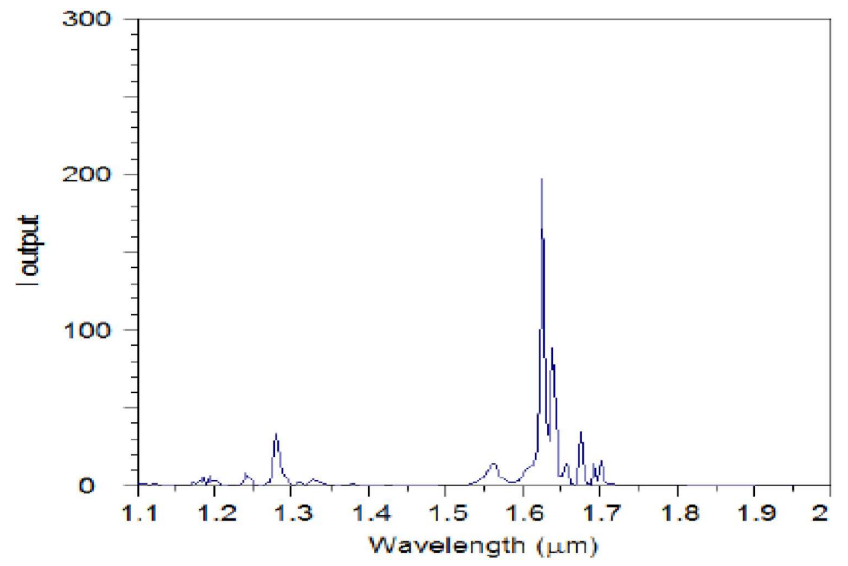

Fig. 5. The spectral response in transmission of the optimized AND logic gate.
The characteristic key of the studied topology is the addition of the holes in the $\mathrm{Y}$-junction center, the radius of the central hole is optimized to increase the power at the output port and to cancel the modal spread in the surface.
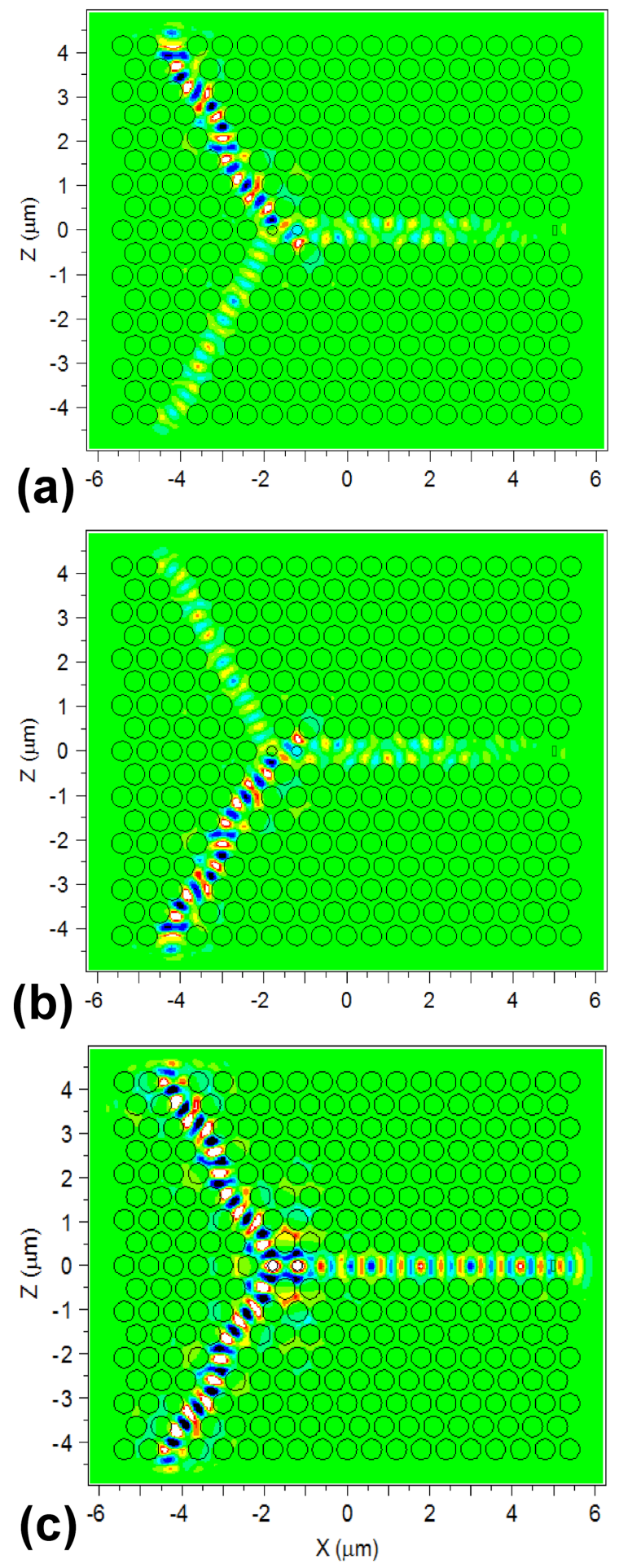

Fig. 6. Field distributions at steady state of the AND logic gate for (a) $\mathrm{A}=1, \mathrm{~B}=0$; (b) $\mathrm{A}=0, \mathrm{~B}=1$ and (c) $\mathrm{A}=1, \mathrm{~B}=1$. 
We note that adding holes at the center of the junction, the mode expansion is suppressed, also the optical volume is then reduced, the mode cannot expand and the excitation of higher order modes is suppressed, resulting in clean and efficient splitting.

The propagation mode is not affected by the accident posed by the corners, allowing the wave to follow the direction of bends. The transmission properties are improved with this configuration and the total transmission at the output ports is improved in comparison with the not optimized structure, this is clearly seen in Fig. 5.

The cavity mode whose magnetic field configuration is depicted in Fig. 6 needs to be coupled to one of the guided modes in the $\mathrm{PC}$ waveguide. The field is computed using 2D FDTD simulations. Among the possible waveguide bands inside the PC band gap.

We first apply continuous wave signal at ports A and B separately and then simultaneously at both input ports $\mathrm{A}$ and $\mathrm{B}$ with input power $P_{i}$.

The output power is $0.017 P_{i}$ for separate excitation with power $P_{i}$ at input port $\mathrm{A}$ as well as for separate excitation at input port B.

For simultaneous excitation with power $P_{i}$ at both input ports $\mathrm{A}$ and $\mathrm{B}$ the output power obtained is $1.038 P_{i}$.

Thus the system performs as an AND gate as summarize in Table $\mathrm{I}$.

TABLE I

The truth table for AND logic gate, where the output signal is as a function of input power $P_{i}$.

\begin{tabular}{c|c|c|c}
\hline \hline Port A & Port B & Logical output & Port C \\
\hline 0 & 0 & 0 & $0 P_{i}$ \\
0 & 1 & 0 & $0.017 P_{i}$ \\
1 & 0 & 0 & $0.017 P_{i}$ \\
1 & 1 & 1 & $1.038 P_{i}$
\end{tabular}

The results illustrated in Fig. 6 prove that the structure suggested could really functioned as a logical gate AND using the devices optic based on photonic crystals, while acting on simple geometrical parameters like the size of the holes.

\section{Conclusion}

In this paper, we studied a two-dimensional photonic crystal in the triangular unit cell by entering proper optimization in order to increase the transmission and obtain a wide bandwidth at the output port. The two dimensional finite-difference time-domain method was employed for the characterization of photonic components.
The evaluation of the transmission spectra for the different components designed has been completed. To reduce the mode expansion at the branching region, we have performed numerical simulations on Y-junction waveguide, and achieved an improvement of transmission by placing two microcavities in the center of the three waveguides. We found through this study that the transmission properties are clearly improved with this configuration, the propagation mode is not affected by the accident posed by the corners, allowing the wave to follow the direction of bends and we have proved that the proposed structure could really function as an AND logic gate with a highest transmission and low losses.

\section{References}

[1] Y. Akahane, T. Asano, B.S. Song, S. Noda, Nature 425, 944 (2003).

[2] W.B. Fraga, J.W.M. Menezes, M.G. da Silva, C.S. Sobrinho, A.S.B. Sombra, Opt. Com. 262, 32 (2006).

[3] T. Fujisawa, M. Koshiba, J. Optic. Soc. Am. B 23, 684 (2006).

[4] D. Jovanović, R. Gajić, D. Djokić, K. Hingerl Acta Phys. Pol. A 116, 55 (2009).

[5] R.K. Sinha, S. Rawal, Optic. Quant. Electr. 40, 603 (2008).

[6] S. Rawal, R.K. Sinha, Opt. Com. 282, 3889 (2009).

[7] A. Taflove, S.C. Hagness, Computational Electrodynamics: The Finite Difference Time Domain Method, 3rd ed., Artech House Publishers, Norwood 2005.

[8] Y. Tetsuro, G. Masahiro, K. Toshiaki, N. Kazuhiro, S. Shinnosuke, IEEE J. Quant. Electr. 38, 37 (2002).

[9] C. Wang, Z. Zhou, Z.Y. Li, Opt. Expr. 19, 26948 (2011).

[10] H. Giden, D. Yilmaz, M. Turduev, H. Kurt, E. Çolak, E. Ozbay, Appl. Phys. Letter 104, 031116 (2014).

[11] C. Lu, X. Hu, Y. Zhang, Z. Li, X. Xu, H. Yang, Q. Gong, Appl. Phys. Lett. 99, 051107 (2011).

[12] A. Kinkhabwala, Z. Yu, S. Fan, Y. Avlasevich, K. Müllen, W.E. Moerner, Nature Photon., 3, 654 (2009).

[13] F. Abdel Malek, W. Belhadj, S. Haxha, H. Bouchriha, J. Lightwave Technol. 25, 3168 (2007). 